Article

\title{
Symmetry in Infancy: Analysis of Motor Development in Autism Spectrum Disorders
}

\section{Gianluca Esposito* and Paola Venuti}

Department of Cognitive Science and Education, University of Trento, via Matteo del Ben 5b, 38068 Rovereto TN, Italy; E-Mail: paola.venuti@unitn.it

* Author to whom correspondence should be addressed; E-Mail: gianluca.esposito@unitn.it.

Received: 27 October 2009 / Accepted: 5 December 2009 / Published: 10 December 2009

\begin{abstract}
Motor asymmetry, defined as the lack of symmetry in movements or postures, is often observed briefly in many typically developing children. However, if such asymmetry persists, it may be a sign of neurological disease. Recent studies have suggested that motor asymmetries may be an early symptom of Autism Spectrum Disorders (ASD). ASD involve a range of social, cognitive, and behavioral problems, at different degrees of functioning, which are thought to be the final common pathway of multiple etiological mechanisms. Furthermore, early identification of ASD has been recognized as a critical aspect for treatment. Our study aims to analyze symmetry in the motor milestones of infants with ASD compared with typically developing infants (TD) or infants with other developmental delay (DD) during the first year of life. Our results highlight that there are different patterns of motor symmetry in the groups. In particular, infants with ASD scored significantly poorer (higher levels of asymmetry) then the TD and DD infants. We also identified two subgroups of infants with ASD, one with a typical level and the other with a lower level of motor functioning. Implications of the study for diagnosis and treatment are described.
\end{abstract}

Keywords: motor symmetry; postural symmetry; Autism Spectrum Disorders

\section{Introduction}

The development of motor autonomy is the infant's main task in the first year of life. The process can be described as a series of distinct stages, where mastering each stage prepares the infant's progression to the next one. At the beginning of each stage, movement is characterized by a persistent 
lack of symmetry. As the stage progresses, movement becomes more and more symmetric, and during each stage complex reflex patterns appear and are integrated with those already existing [1]. The main stages of motor development, often defined as "milestones," are the following: (i) lying; (ii) rolling; (iii) sitting; (iv) crawling; (v) standing; and (vi) walking. One qualification: although this is the typical progression, we should note that occasionally some infants appear to skip stages with no negative consequences.

Lying is an active posture, even in the first few days of life. As has been highlighted by Casaer [2], newborns maintains specific active postures while lying. Lying can also be seen as the first occasion for infants to test their vestibular system. Indeed, in typical infants a general symmetry, defined as similarity in the position of the two sides of the body, can be seen [3].

Rolling. From the first months of life, although there is considerable variability, infants show the "rolling" movement. This movement is extremely important for the infant's increasing strength and coordination. Two patterns can be seen: rolling from front to back and rolling from back to front. For some typically developing infants, this pattern may not emerge until seven or eight months of age.

Sitting. Typically developing infants are able to sit without external support, on average, at about seven to eight months. This movement requires integration among the vestibular centers and the muscles that control the trunk and hips. Although the sitting posture is usually achieved during the second half of the first year of life, its emergence is a continuous process. Indeed, during the first two months, an infant's muscles have not yet developed adequate strength to sit. During the third month, infants usually are able to hold their heads in line with their bodies (although only for short periods of time because the neck muscles tire). After the fifth month infants can hold their heads steady for longer periods of time, which is a very important developmental step-by being able to look about for longer period of time, infants can begin to explore their environment in more depth. At about six to seven months of age, typically developing infants begin to show the righting reflex. This reflex, which is shared with other nonhuman primates, is the ability to resume an optimal position when there has been a departure from it. For example, in the case of sitting, when infants are held around the chest and their bodies are tilted, they extend their arms in an attempt to keep balance. After the activation of this reflex, infants develop several skills that provide the opportunity to sit while engaging in other activities.

Crawling. Often infants start to crawl at about the same age they begin to sit. Crawling is a specific four-beat gait involving both hands and knees. In a typical crawl, first the hand is extended, followed by the diagonal knee, the other hand, and finally the diagonal knee. This is the first gait most humans learn, and although its use is largely limited to early childhood, it is an important milestone [4].

Standing. The ability to stand usually emerges around the tenth month of life. It represents perhaps the most important motor skill infants learn during their first year of life. Once able to stand, infants have the opportunity to observe the environment from a totally different prospective. The world can be seen through a larger visual field and the concept of prospective and distance can more easily be experienced.

Walking. Most babies learn to walk between twelve and fifteen months of age. In the general population, this age is normally distributed with a standard distribution of almost three months, which means that a child who is not yet walking by eighteen months of age may still be regarded as typically developing. The reflexes that form the foundation for walking are probably innate. However, children 
cannot walk until their muscles have grown and developed enough strength to sustain their body weight.

A consistent developmental pattern characterizes all of the motor developmental milestones just described: all are performed at the beginning in a very asymmetric way (i.e., a lack of balance or "patterned self-similarity" among the position of the limbs). Then, as the motor expertise of the child progresses and the muscles become stronger, the movement is produced in a more symmetrical fashion. This means that a general degree of asymmetry is always present in the execution of a motor pattern, at least in the early stage of development of that specific pattern. Although the value of early asymmetry as a sign of neurological disease is controversial, indeed asymmetry is part of a wide range of patterns shown by typical infants [5,6], and a number of studies have highlighted how persistent asymmetry can indicate abnormalities associated with developmental disorders [3,7].

\subsection{Persistent Asymmetry and Autism Spectrum Disorder}

Autism is a lifelong developmental condition characterized by impaired social interaction and communication as well as repetitive behaviors and restricted interests [8]. The manifestation of autism varies in severity, and it is generally agreed that there is a spectrum of disorders (Autism Spectrum Disorder, ASD). A number of studies have pointed out that early assessment can lead to a substantial improvement in the life conditions of children with autism. According to these studies, early diagnosis and the study of predictors of the syndrome are extremely important. Movement has gained much attention in the last decades as an early indicator of ASD. Movement disorders are considered one of the first signs that may precede social or linguistic abnormalities [9]. In particular, authors have focused their work on fine and general movements, and walking and spinning (for a review see [10]).

\subsection{Motor Developmental Milestones and ASD}

Based on the hypothesis that movement may be an early indicator of ASD, several studies have attempted to describe motor developmental milestones in children with ASD as opposed to Typically Developing (TD) children. These studies have investigated (i) lying, (ii) rolling, (iii) sitting, (iv) crawling, (v) standing, and (vi) walking.

Lying. A number of studies have shown that persistent deviations from the normal patterns of lying can indicate abnormalities associated with ASD [1,3,7]. For example, in one study [7] persistent asymmetries at the age of four months in infants (later diagnosed with ASD) lying on their stomachs were shown.

Rolling. Impairments in rolling in children with ASD have been described [7]. They note that some children cannot perform rolling at all, while some others can begin a rotation pattern but are unable to complete the motor sequence. Furthermore, even children with ASD who were able to perform the complete motor sequence showed quite a different pattern from the pattern described in TD children.

Sitting. In their 1998 study Teitelbaum and colleagues showed that children with ASD at six months of age were not able to maintain sitting stability, whereas typically developing children of this age usually can. Moreover, of the children with ASD who were able to maintain a sitting position, their posture was persistently asymmetrical; specifically, their weight was often not distributed equally on both sides. Some of the children analyzed in this study were still unable to sit independently as late as 
10 months of age. Interestingly, in both studies [1,7] Teitelbaum and colleagues have shown that infants later diagnosed with ASD during the first year of life displayed a pattern of falling forward or backward from a sitting position without using protective reflexes of the arms and verticalization of the head (parachute reflex).

Crawling. In 2004, Teitelbaum and colleagues [1] showed that some infants later diagnosed with ASD exhibited crawling patterns that deviated from the basic diagonally opposing limb patterns. In particular, infants with ASD exhibited a pattern of asymmetrical crawling.

Standing. Very few data are reported about standing in infants with ASD during the first year of life. An exception is a case, described by Teitelbaum and colleagues [7], of akinesia (a signal of motor disorder) in a girl later diagnosed with ASD who "stood in one place leaning her back against a heavy piece of furniture for periods as long as 15 minutes at a time”.

Walking. Walking represents the most investigated of the motor milestones in children with ASD. The first studies on walking and ASD were conducted in the late 1970s. Damasio and Maurer [11] showed that children with ASD between three and ten years of age walk somewhat like Parkinsonian adults in that they walk more slowly and with shorter steps than typically developing children. Nonetheless, the existence of such a Parkinsonian-type disturbance was disputed by Hallett et al. [12]. Allen and Courchesne [13], using functional Magnetic Resonance, found that certain areas of the cerebellar vermis are incompletely developed in children with autism. Other studies have stressed the importance of the dopaminergic system and of the basal ganglia [14-16]. This also supports the view that movement disorders might play a role in ASD.

Although there is agreement on the presence of motor disturbances in autism in older ages, a general consensus on the existence of early motor developmental disorders in autism still lacks. While some independent studies $[1,10,17,18]$ have described differences in motor development of ASD, other studies $[19,20]$ have been unable to replicate these findings and in particular to identify differences between ASD and children with other developmental disorders. Among the studies that have considered worth investigating motor development in ASD in attempts to identify early markers, the majority have focused on lying and walking.

In sum, the aim of this study is to analyze symmetry in the motor milestones of children with ASD compared with typically developing children (TD) and children with other developmental delay (DD) during the first year of life. The purpose is to attempt to identify early markers of the syndrome. In particular, we describe two studies that analyze symmetry during sitting and standing.

\section{Method}

\subsection{Participants}

Frames captured from retrospective home videos taken when infants were sitting at 5-7 months of age ( $\mathrm{ASD}=10 ; \mathrm{TD}=10 ; \mathrm{DD}=10)$ and when infants were standing at 9-11 months of age (ASD = 12; $\mathrm{TD}=12$; $\mathrm{DD}=12$ ) were analyzed. Participants were recruited from the clinical population of the Observation and Functional Diagnosis lab (ODFLab) at the University of Trento. ODFLab is a referral center for developmental disabilities, and children and adolescents are brought from across northern Italy for assessment and treatment. The children belonging to the ASD group received their diagnoses 
at ages when autism can be reliably diagnosed (about 36 months of age). Multiple diagnostic criteria were used to confirm the presence of autism. Each child (a) had been diagnosed with autistic disorder prior to referral to the study, (b) received a current clinical diagnosis of autistic disorder according to Diagnostic and Statistical Manual of Mental Disorders (DSM-IV-TR; American Psychiatric Association [8]) criteria, and (c) met all criteria for autism on the Autism Diagnostic Observation Schedule-ADOS [21]. A group of children with developmental delays was recruited to provide both chronological and developmental age matching for the autism group. None had a current or previous clinical or DSM-IV-TR diagnosis of autistic disorder or pervasive developmental disorder-not otherwise specified. A group of typically developing children was also recruited as a comparison to provide chronological age matching; these children had normal hearing and vision and did not present any significant medical or developmental concerns.

\subsection{Procedure}

General Procedure. Since the purpose of the study was to analyze behavior that occurred early in life, before it is possible to diagnose ASD reliably, a retrospective approach was employed. Although retrospective studies based on home videos have a number of methodological problems (e.g., difficulty in controlling variables such as the infant's age and the length, variability of content, and structure of the video segments), they provide access to very early periods in development, months or years before a child with ASD is diagnosed [22,23]. In addition to an ASD group, two comparison groups were recruited: children with typical development (TD) and children with developmental delay (DD) but not ASD.

Slideshow Preparation. Once families with home videos taken when their children were less than one year of age were selected, as described subsequently, each video was screened for minimum quality standards. Any tapes not already in standard AVI format were converted. Based on the child's birth date and the date of the video, an age (in weeks) was assigned each video. A research assistant who was not aware of the goals of the studies edited the videos. The research assistant was instructed to select from the 21 hours of videos recruited, all scenes from the available footage during which the child was sitting (or trying to sit) or was standing (or trying to stand), and during which the child's whole body was visible. This resulted in an average of two sitting and two standing scenes per child. Four frames for each second were randomly selected from these scenes (the initial video had 25 frames per second). The frames for each child were grouped together, assigned a serial number, and organized into two slideshows (one for sitting and another for standing). A total of 212 frames from $1320 \mathrm{~s}$ of materials were analyzed (sitting= $600 \mathrm{~s}$; standing $=720 \mathrm{~s}$ ).

Coding. Two coders coded the sitting frames and another two coders the standing frames. Frames were coded as either symmetric or not based on prototypic symmetric patterns we developed, the Positional Pattern for Symmetry during Sitting (PPSSit) and the Positional Pattern for Symmetry during Standing (PPSStand). We defined these patterns using Eshkol-Wachman Movement Notation (EWMN) [24], which uses a simplified, general form to represent bodies as stick figures. Each limb, as many as desired, is reduced to its longitudinal axis, an imaginary straight line of unchanging length. The movements of a single limb are then represented with respect to an imaginary sphere; for example, when marking off intervals of 45 degrees, eight positions are obtained (for additional details see 
http://en.wikipedia.org/wiki/Eshkol-Wachman_Movement_Notation). Based on EWMN, the PPSSit and the PPSStand define all possible symmetric positions of a body in a sitting and standing position, respectively. A body is regarded as symmetric when the position of corresponding limbs (an arm and the other arm, a leg and the other leg) is the same (using $45^{\circ}$ accuracy). Specifically, the PPSSit and the PPSStand define 20 possible symmetric positions for the arms and 4 for the legs [see 3], giving a total of 80 symmetrical positions for the whole body. The sitting coders then compared each sitting frame to the 80 prototypical symmetric sitting positions and coded it as symmetric if a matching prototype was found (see Figure 1); likewise for the standing coders and frames (see Figure 2). After all frames were coded, the percentage of sitting and standing frames coded symmetric was calculated for each participant. Thus, our dependant measures were the proportion of frames showing asymmetry for each infant for sitting and for standing.

Two coders, blind to the hypotheses of the study, were trained to use the PPSSit and another two, likewise blind, to use the PPSStand. Training was accomplished through the study of the EWMN and the PPSSit or PPSStand manual, followed by viewing of video samples of children not being used in the study and a focus group discussion with an expert on both the EWMN and the PPSSit or PPSStand. Interrater reliability was calculated on $40 \%$ of the sample using Cohen $\mathrm{k}$ [25], which gave statistically acceptable kappas of 0.85 and 0.76 for sitting and standing, respectively.

Figure 1. For the purpose of assessing symmetry during sitting, each frame, that shows an image of the infant sitting, is compared to symmetrical position of the PPSSit. (a) When the position of the body in the frame correspond to a symmetric (symmetrical position of the body is a position where similarity in relative position of corresponding limbs -an arm and the other arm, a leg and the other leg- is showed with an accuracy of $45^{\circ}$ ) position, the frame is coded as symmetric. (b) When the position of the body in the frame is not symmetrical, the frame is coded as non-symmetric.
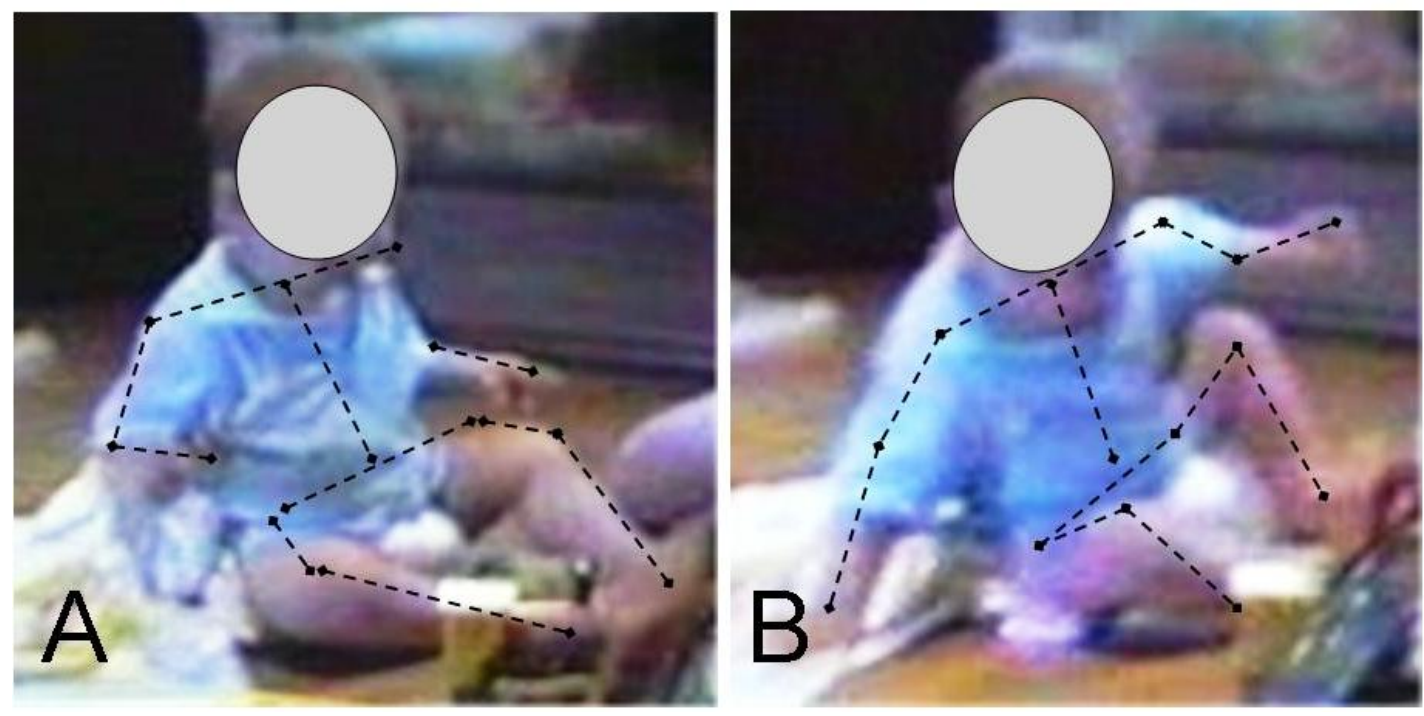
Figure 2. For the purpose of assessing symmetry during standing, each frame, that shows an image of the infant standing, is compared to symmetrical position of the PPSStand. (a) When the position of the body in the frame correspond to a symmetric (symmetrical position of the body is a position where similarity in relative position of corresponding limbs -an arm and the other arm, a leg and the other leg- is showed with an accuracy of $45^{\circ}$ ) position, the frame is coded as symmetric. (b) When the position of the body in the frame is not symmetrical, the frame is coded as non-symmetric.
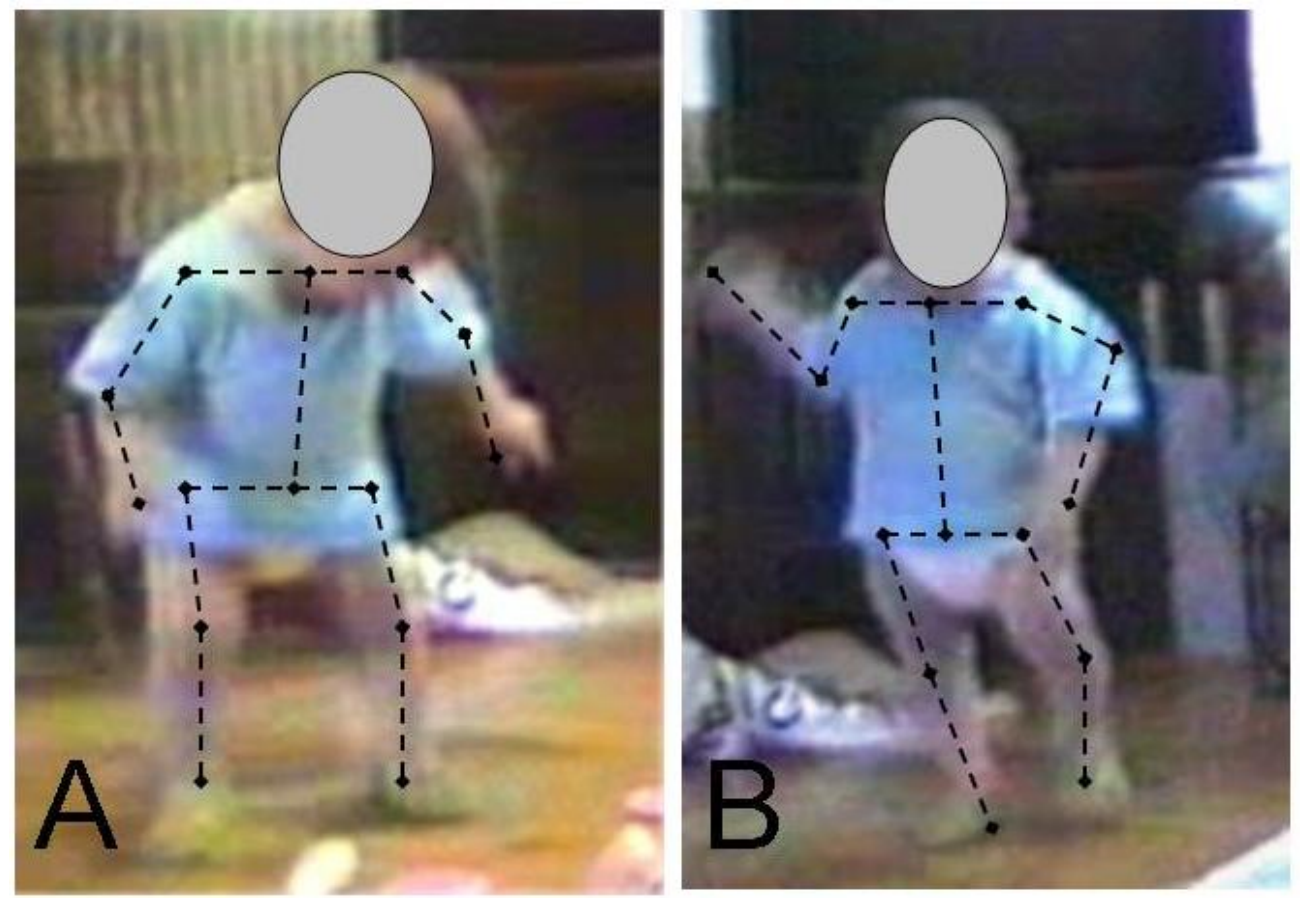

\section{Results}

\subsection{Symmetry while sitting}

The level of symmetry showed significant differences among the groups (ASD: $\mathrm{M}=40.4$ $\mathrm{SD}=19.8 ; \mathrm{DD}: \mathrm{M}=52.6 \mathrm{SD}=8.1$;D: $\mathrm{M}=49.5 \mathrm{SD}=6.8 ; \mathrm{F}(2,30)=4.12, \mathrm{p}<0.05)$. Then, considering the higher variability of infants with ASD, the symmetry scores were subjected to cluster analysis [26]. Employing the KMeans cluster analysis (person approach), infants were empirically identified as belonging to one of two clusters: (1) lower level of symmetry (LLS; $n=6$ ) or (2) higher level of symmetry (HLS; $\mathrm{n}=24$ ). Significant differences were found for level of symmetry $(F(1,30)=35.23, p<0.001)$. All the participants in the LLS cluster belonged to the ASD group. The ASD group was therefore split in two subgroups: (a) ASD with lower level of symmetry ( $\mathrm{n}=6$, symmetry level mean $=33.5$ and SD = 7.3; (b) ASD with higher level of symmetry ( $n=4$, symmetry level mean $=48.6$ and SD $=5.9$ ). These ASD subgroups were statistically different using Student's t-test $(\mathrm{t}=6.78, \mathrm{df}=10, \mathrm{p}<0.05)$. Results are shown in Figure 3. 
Figure 3. Descriptive statistics of the level of symmetry during sitting in the ASD, DD and TD groups. $* \mathrm{p}<0.05$.

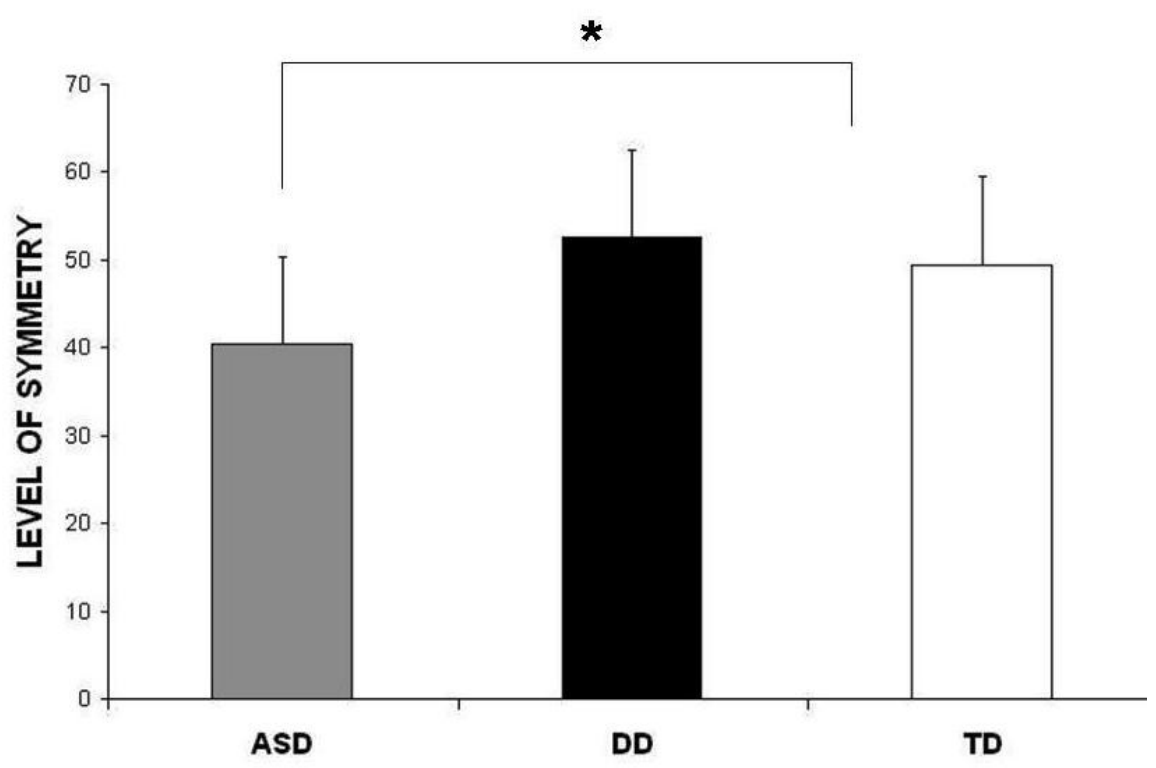

\subsection{Symmetry while standing}

The level of symmetry showed no significant differences among the groups (ASD: $\mathrm{M}=61.4$ $\mathrm{SD}=16.3$; $\mathrm{DD}: \mathrm{M}=64.5 \mathrm{SD}=14.8$; $\mathrm{TD}: \mathrm{M}=59.3 \mathrm{SD}=11.9 ; \mathrm{F}(2,36)=1.29, \mathrm{p}=\mathrm{ns})$. Then the symmetry scores were subjected to cluster analysis [22]. Employing the KMeans cluster analysis (person approach), infants were empirically identified as belonging to one out of the two clusters: (1) lower level of symmetry (LLS; $n=16$ ); (2) higher level of symmetry (HLS; $n=20$ ). Significant differences were found for level of symmetry $(\mathrm{F}(1,36)=14.9, \mathrm{p}<0.01)$. The two clusters pooled together children of the three groups (LLS = 7 ASD, 5 DD, 6 TD; HLS = 5 ASD, 7 DD, 6 TD).

\section{Discussion and Conclusions}

The goal of this investigation was to expand previous studies present on the literature of early motor development in ASD. In particular, two studies were proposed to analyze two developmental motor milestones: "sitting" and "standing". We hypothesized that movement analysis could show differences in the motor milestones of infants with ASD compared to those with TD or with DD. In particular, we expected to find more asymmetric motor pattern and posture while sitting and standing in children with ASD.

Data analysis showed differences among the AD, TD and DD groups for sitting but not for standing. With respect to sitting, and in agreement with previous studies run in other laboratories [1], our results demonstrated that between five and seven months of age different patterns in movement among the groups are evident. In particular, children with a diagnosis of ASD showed more asymmetric posture. Regarding internal variability, the standard deviation for the ASD group was much larger than that for the TD and DD groups. Furthermore, cluster analysis on the whole group of participants showed two clusters (LLS and HLS) representing two levels of mastering the own body in a symmetric position, and the lower level group was totally composed of children with ASD. Thus, as 
far as symmetry during sitting is concerned the ASD group shows a bi-modal distribution. This finding could be of some clinical value, since the different subgroups could be related to multiple etiological and neuropathological mechanisms that lead to ASD. Indeed the autism spectrum suggests that different neuronal pathways does exist [27].

The lack of differences for standing might have been predicted. Although there is one report of motor anomalies in children with ASD while standing in the first year of life, this report was based on the observation of a single girl with ASD [7]. We think that the analysis of standing between nine and eleven months of age did not show differences because all three groups showed low levels of symmetry. In fact is it possible that all children in this developmental stage (9-11 months) are learning how to stand and maintain balance and for this reason they show high levels of asymmetry. This result also agrees with the idea that at the beginning of each developmental motor milestone movement is characterized by a persistent lack of symmetry. Probably standing, which is compromised in older children with ASD [28], is not a useful marker during the first year of life.

In general, these results are in agreement with evidence that acknowledges the importance of some specific motor milestones (such as lying, sitting, crawling, rolling and walking) as an early indicator for a diagnosis of ASD. Furthermore, these results agree with the idea that there are some specific neural mechanisms, implied in motor control, which play a role in the motor anomalies shown by a subgroup of children with ASD. In some previous studies [3,10] a Purkinje cells disruption has been proposed as one possible mechanism that compromises motor development in ASD. Indeed general agreement exists that the microscopic anatomic pathology of ASD involves Purkinje cell loss and neuron size reductions in the cerebellum. Furthermore, in a report by Kern [29] it is speculated some children may become ASD from neuronal cell death or brain damage occurring postnatally due to injuries. Purkinje cells in the cerebellum can be selectively vulnerable to certain types of insult (e.g., hypoxia, excitotoxicity [30]) and they may play a role in motor disturbances in children with ASD.

A limitation of this study that may have affected the statistical power of the analyses is the small sample size. However, it is difficult to recruit large samples for retrospective studies. In particular, retrospectives home videos studies are even harder to mount since parents may not have videos of the child or may not have videos of the specific age being analyzed. Although the study used a small sample, the results provide preliminary insights for future studies.

Future studies should indeed further investigate other motor developmental milestones of ASD, such as crawling and rolling. Such investigations may reveal possible biomarkers for early assessment of ASD that could be useful for diagnostic purposes. Particularly, future research needs to include bigger samples of children with ASD so as to facilitate post hoc analyses that investigate possible subgroups among children with Autism Spectrum Disorder.

\section{Acknowledgements}

All the participants who participated in this study are gratefully acknowledged. Gratitude is expressed to Consuelo Scalzeri (ODFLab, University of Trento) for her research assistance and to Roger Bakeman (Georgia State University) for reading and commenting on the paper. 


\section{References and Notes}

1. Teitelbaum, O.; Benton, T.; Shah, P.K.; Prince, A.; Kelly, J.L.; Teitelbaum, P. Eshkol-Wachman Movement Notation in diagnosis: the early detection of Asperger's syndrome. Proc. Natl. Acad. Sci. 2004, 101, 11909-11914.

2. Casaer, P. Postural Behavior in Newborn Infants; William Heinemann Medical Books: London, UK, 1979.

3. Esposito, G.; Venuti, P.; Maestro, S.; Muratori, F. Movement in infants with Autism Spectrum Disorder: The Analysis of Lying. Brain Dev. 2009, 31, 131-138.

4. Freedland, R.L.; Bertenthal, B.I. Developmental changes in interlimb coordination - transition to hands-and-knees crawling. Psychol. Sci. 1994, 5, 26-32.

5. Cioni, G.; Duchini, F.; Milianti, B.; Paolicelli, P.B.; Sicola, E.; Boldrini, A.; Ferrari, A. Differences and variation in the patterns of early independent walking. Early Hum. Dev. 1993, 35, 193-205.

6, Cioni, G.; Bos, A.F.; Einspieler, C.; Ferrari, F.; Martijn, A.; Paolicelli, P.; Rapisardi, G.; Roversi, M.F.; Prechtl, H.F.R. Early neurological signs in preterm infants with unilateral intraparenchymal echodensity. Neuropediatrics 2000, 31, 240-251.

7. Teitelbaum, P.; Teitelbaum, O.; Nye, J.; Fryman, J.; Maurer, R. Movement analysis in infancy may be useful for early diagnosis of autism. Proc. Natl. Acad. Sci. USA 1998, 95, 13982-13987.

8. Diagnostic and statistical manual of mental disorders, 4th Ed.; American Psychiatric Association: Washington, DC, USA, 2000.

9. Leary, M.; Hill, D. Moving on: Autism and movement disturbance. Ment Retard 1996, 34, 39-53.

10. Esposito, G.; Venuti, P. Movement and Autistic Disorder: the analysis of gait after 6 months of independent walking. Percept. Mot. Skills 2008, 106, 259-269.

11. Damasio, A.R.; Maurer, R.G. A neurological model for childhood autism. Arch. Neurol. 1978, 35, 777-786.

12. Hallett, M.; Lebiedowska, M.K.; Thomas, S.L.; Stanhope, S.J.; Denckla, M.B.; Rumsey, J. Locomotion of autistic adults. Arch. Neurol. 1993, 50, 1304-1308.

13. Allen, G.; Courchesne, E. Differential effects of developmental cerebellar abnormality on cognitive and motor functions in the cerebellum: an fMRI study of autism. Am. J. Psych. 2003, 160, 262-273.

14. Vernazza-Martin, S.; Martin, N.; Vernazza, A.; Lepellec-Muller, A.; Rufo, M.; Massion, J.; Assaiante, C. Goal Directed Locomotion and Balance Control in Autistic Children. J. Autism Dev. Disord. 2005, 35, 91-102.

15. Rinehart, N.J.; Tonge, B.J.; Iansek, R.; McGinley, J.; Brereton, A.V.; Enticott, P.G.; Bradshaw, J.L. Gait function in newly diagnosed children with autism: cerebellar and basal ganglia related motor disorder. Dev. Med. Child. Neurol. 2006, 48, 819-824.

16. Esposito, G.; Venuti, P. Gait Analysis in children with autism spectrum disorder and in children with typical development. Gait Post. 2006, 24S, 269-270.

17. Iverson, M.J.; Wozniak, R.H. Variation in vocal-motor development of infant siblings of children with autism. J. Autism Dev. Disord. 2007, 37, 158-170. 
18. Loh, A.; Soman, T.; Brian, J.; Bryson, S.E.; Roberts, W.; Szatmari, P.; Smith, I.M.; Zwaigenbaum, L. Stereotyped Motor Behaviors Associated with Autism in High-risk Infants: A Pilot Videotape Analysis of a Sibling Sample. J. Autism Dev. Disord. 2007, 37, 25-36.

19. Provost, B.; Lopez, B.R.; Heimerl, S. A Comparison of Motor Delays in Young Children: Autism Spectrum Disorder, Developmental Delay, and Developmental Concerns. J. Autism Dev. Disord. 2007, 37, 321-328.

20. Ozonoff, S.; Young, G.S.; Goldring, S.; Greiss-Hess, L.; Herrera, A.M.; Steele, J.; Macari, S.; Hepburn, S.; Rogers, S.J. Gross motor development, movement abnormalities, and early identification of autism. J. Autism Dev. Disord. 2008, 38, 644-656.

21. Lord, C.; Risi, S.; Lambrecht, L.; Cook, E.H.; Leventhal, B.L.; DiLavore, P.C.; Pickles, A.; Rutter, M. The Autism Diagnostic Observation Schedule-Generic: A standard measure of social and communication deficits associated with the spectrum of autism. J. Autism Dev. Disord. 1989, 30, 205-223.

22. Baranek, G.T. Autism during infancy: a retrospective video analysis of sensory-motor and social behaviours. J. Autism Dev. Disord. 1999, 29, 213-224.

23. Werner, E.; Dawson, G.; Osterling, J.; Dinno, N. Brief report: Recognition of autism spectrum disorder before one year of age: A retrospective study based on home videotapes. J. Autism Dev. Disord. 2000, 30, 157-162.

24. Eshkol, N.; Harris, J. Eshkol Wacheman Movement Notation Part 1; The Movement Notation Society: Holon, Israel, 2001.

25. Cohen, J. Weighted kappa: Nominal scale agreement with provision for scaled agreement or partial credit. Psychol. Bull. 1968, 70, 213-220.

26. Hartigan, J.A.; Wong, M.A. A K-means clustering algorithm. Appl. Stat. 1979, 28, 100-108.

27. Gillberg, C. Neurodevelopmental processes and psychological functioning in autism. Dev. Psychopathol. 1999, 11, 567-587.

28. Molloy, C.A.; Dietrich, K.N.; Bhattacharya, A. Postural Stability in Children with Autism Spectrum Disorder. J. Autism Dev. Disord. 2003, 33, 643-652.

29. Kern, K.J. Purkinje cell vulnerability and autism: a possible etiological connection. Brain Dev. 2003, 25, 377-382.

30. Welsh, J.P.; Yuen, G.; Placantonakis, D.G.; Vu, T.Q.; Haiss, F.; O’Hearn, E. Why do Purkinje cells die so easily after global brain ischemia? Aldolase C, EAAT4, and the cerebellar contribution to posthypoxic myoclonus. Adv. Neurol. 2002, 89, 331-359.

(C) 2009 by the authors; licensee Molecular Diversity Preservation International, Basel, Switzerland. This article is an open-access article distributed under the terms and conditions of the Creative Commons Attribution license (http://creativecommons.org/licenses/by/3.0/). 